\title{
Subclinical hypercortisolism: debatable or visible on the lightbox?
}

\author{
Dimitra A. Vassiliadi · Stylianos Tsagarakis
}

Published online: 16 May 2012

(C) Springer Science+Business Media, LLC 2012

Adrenal nodules unrelated to clinically overt adrenal disease became visible more than 120 years ago ever since the first autopsy studies [1]. Nowadays, through the development of modern radiology modalities, adrenal nodules previously identified only at autopsy or surgery, are easily visible during imaging of the abdomen. The importance of this for the practicing endocrinologist is highlighted by the fact that hardly a week goes by without a patient being referred for further evaluation of an incidentally depicted adrenal mass. Despite, however, the high prevalence of adrenal incidentalomas, about $4 \%$ in the middle-age population, approaching $7 \%$ in the elderly ones [2,3], and an ongoing stream of published papers, its relevance for the patient remains in many occasions largely inconclusive.

The vast majority of adrenal incidentalomas are benign adrenocortical adenomas although rare entities, such as myelolipomas, cysts, ganglioneuromas, onconcytomas, may also be encountered. A significant proportion of patients, about $15 \%$, have bilateral lesions [4]. Since most adrenal incidentalomas arise from adrenal tissue, a substantial proportion of them are functional in terms of autonomous production of various adrenal hormones, including cortisol, aldosterone, catecholamines or rarely androgens. Subclinical hypercortisolism ( $\mathrm{SH}$ ), defined by an abnormal endocrine work-up indicating autonomous cortisol secretion in the absence of obvious clinical stigmata of Cushing's syndrome, is by far the most commonly encountered abnormality. It is detected in a varying proportion of subjects, between 1 and $47 \%$, depending on the diverse criteria used to define it [5]. The finding of an adrenal mass undoubtedly raises concerns

D. A. Vassiliadi · S. Tsagarakis $(\bowtie)$

Department of Endocrinology, Diabetes and Metabolism, Evangelismos Hospital, 45 Ipsilantou St, 10676 Athens, Greece e-mail: stsagara@otenet.gr about the possibility of malignancy but adrenocortical cancer in this context is rare, especially when the size of the lesion is less than $4.0 \mathrm{~cm}$, as is the case with most adrenal incidentalomas [6]. Thankfully, differentiation between benign and malignant adrenal lesions is visible with much accuracy with current imaging techniques. On CT scanning, which is the modality of choice for evaluating the adrenal glands, adrenocortical adenomas are well-circumscribed ovoid masses with typically low attenuation values (less than $10 \mathrm{HU}$ ) on unenhanced images, due to the high content in lipids. The $20-30 \%$ of lipid-poor adrenal adenomas that exhibit higher attenuation values can be further evaluated either using contrast washout techniques or MRI scans. Benign adenomas on $\mathrm{CT}$ show an absolute and a relative percentage of contrast washout of more than 60 and $40 \%$, respectively. MRI may provide additional information for the few remaining indeterminate lesions, using $\mathrm{T} 1$-weighted, T2-weighted, and especially chemical shift imaging (CSI); benign adenomas are homogenous, have low or equal signal intensity compared to the liver on T2-weighted images and demonstrate significant signal intensity loss on out-of-phase CSI compared to the in-phase images. Thus, radiology has a major role in identifying the rare cases of malignancy among adrenal incidentalomas through the initial imaging phenotype combined with a repeat scan to evaluate potential rapid and significant increase in size.

In this Endocrine issue Olsen et al. [7] in a multicentre, prospective study attempt to expand the usefulness of imaging visibility toward a functional perspective. The authors investigated 235 patients presenting with benign appearing incidentally detected adrenal masses and correlated CT findings such as unenhanced attenuation value, size, and bilaterality with the hormonal work-up for the presence of SH. Previous observational data have shown a consistent relationship between the size of the adenoma 
and the degree of autonomous cortisol secretion [8]. More recently we reported that bilateral incidentalomas exhibit SH more frequently than their unilateral counterparts [4]. The authors confirm these findings but the novelty of this study is that the probability of SH also relates inversely to the unenhanced CT attenuation values of the adrenal lesions. Steroid producing cells are characterized by high intracellular lipid content due to the presence of steroid hormones and its precursors, mainly cholesterol. Low attenuation values reflect this lipid-rich nature of steroid cells. But is it possible for an image to also capture hormonal activity by rendering it into a visible entity?

Patients with the lowest attenuation values exhibited SH more frequently (between 40 and $45 \%$ depending also on the size of the adenoma) but the remaining 55-60\% did not. Thus, demonstrating signs for the presence of a steroid production machinery in a cell does not necessarily mean that steroids are also secreted and, more importantly, in an autonomous manner. So, if the presence of autonomous cortisol secretion cannot be viewed based on imaging characteristics, is it possible to exclude it on the same basis? The presented data indicate that even lipid-poor adenomas may harbor $\mathrm{SH}$, in a much lower, albeit not negligible, proportion of $10 \%$, especially if the lesion is larger than $1.8 \mathrm{~cm}$.

The main controversial issue, in this paper, and other similar publications, is the definition of SH [8-10]. Currently $\mathrm{SH}$ is one of the most debatable entities in endocrinology mainly because its diagnosis cannot be made against an indisputable standard. Although $\mathrm{SH}$ has been associated with the presence of non-specific signs of hypercortisolism, these patients lack, by definition, the more specific clinical stigmata of overt Cushing's syndrome, precluding a clinical diagnosis. Therefore, diagnosis is based on an endocrine work-up comprising of the tests used for the diagnosis of overt Cushing's syndrome, including increased midnight serum or salivary cortisol levels, increased urinary free cortisol levels and non-suppression of cortisol levels after dexamethasone administration. The most widely used and easy to perform test is the $1 \mathrm{mg}$ overnight dexamethasone suppression test (DST). However, its sensitivity and specificity varies according to the selected cut-off level. In this study the authors applied a strict cut-off of $1.8 \mu \mathrm{g} / \mathrm{dl}$ that decreases the specificity of DST leading to a very high incidence of abnormal results. To overcome this shortcoming the authors incorporated a low ACTH level as an additional positive test to support the diagnosis of SH. ACTH levels, however, were available for only 142 out of 235 patients whereas other screening tests were not considered making the diagnosis of SH more questionable than already is.
Despite its limitations this study is the first one to attempt foreseeing functionality of an adrenal mass by glancing on the lightbox or, nowadays, the computer screen. Although the main conclusion is that CT appearance does not preclude the presence of $\mathrm{SH}$, it provides some clues on which patients are more likely to harbor $\mathrm{SH}$; those with larger adenomas, bilateral and with low attenuation values are more likely candidates. Taken together, imaging can provide some indication of the presence of intracellular steroids in an adrenal adenoma but demonstrating autonomous cortisol hypersecretion is a clinical decision guided by an elaborate endocrine testing, which already has its pitfalls. In current practice, defining SH is a major controversial issue corroborating Oscar Wilde's phrase in The Picture of Dorian Gray that "mystery is on the visible not on the invisible".

\section{References}

1. M. Letulle, Note sur la degenerescence graisseuse de la capsule surrenale. Bull. Mem. Soc. Anat. Paris 3, 264-267 (1889)

2. R.T. Kloos, M.D. Gross, I.R. Francis, M. Korobkin, B. Shapiro, Incidentally discovered adrenal masses. Endocr. Rev. 16, 460-484 (1995)

3. D.A. Vassiliadi, S. Tsagarakis, Endocrine incidentalomaschallenges imposed by incidentally discovered lesions. Nat. Rev. Endocrinol. 7, 668-680 (2011)

4. D.A. Vassiliadi, G. Ntali, E. Vicha, S. Tsagarakis, High prevalence of subclinical hypercortisolism in patients with bilateral adrenal incidentalomas: a challenge to management. Clin. Endocrinol. (Oxf) 74, 438-444 (2011)

5. S. Tsagarakis, D. Vassiliadi, N. Thalassinos, Endogenous subclinical hypercortisolism: Diagnostic uncertainties and clinical implications. J. Endocrinol. Invest. 29, 471-482 (2006)

6. T.J. Cawood, P.J. Hunt, D. O'Shea, D. Cole, S. Soule, Recommended evaluation of adrenal incidentalomas is costly, has high false-positive rates and confers a risk of fatal cancer that is similar to the risk of the adrenal lesion becoming malignant; time for a rethink? Eur. J. Endocrinol. 161, 513-527 (2009)

7. H. Olsen, E. Nordenstrom, A. Bergenfelz, U. Nyman, S. Valdemarsson, E. Palmqvist, Subclinical hypercortisolism and CT appearance in adrenal incidentalomas: a multicenter study from Southern Sweden. Endocrine (2012). doi:10.1007/s12020-0129622-2

8. S. Tsagarakis, C. Roboti, P. Kokkoris, V. Vasiliou, C. Alevizaki, N. Thalassinos, Elevated post-dexamethasone suppression cortisol concentrations correlate with hormonal alterations of the hypothalamo-pituitary adrenal axis in patients with adrenal incidentalomas. Clin. Endocrinol. (Oxf) 49, 165-171 (1998)

9. M. Terzolo, A. Pia, G. Reimondo, Subclinical Cushing's syndrome: definition and management. Clin. Endocrinol. (Oxf) 76, 12-18 (2012)

10. P.M. Stewart, Is subclinical Cushing's syndrome an entity or a statistical fallout from diagnostic testing? Consensus surrounding the diagnosis is required before optimal treatment can be defined. J. Clin. Endocrinol. Metab. 95, 2618-2620 (2010) 